\title{
Damage detection of composite milling process by recurrence plots and quantifications analysis
}

\author{
Krzysztof Kecik $^{1} \cdot$ Krzysztof Ciecielag $^{1} \cdot$ Kazimierz Zaleski $^{1}$
}

Received: 18 October 2015 / Accepted: 13 June 2016 / Published online: 25 June 2016

(C) The Author(s) 2016. This article is published with open access at Springerlink.com

\begin{abstract}
We study the dynamics of a milling process of carbon fiber reinforced plastic based on experimental time series of cutting force with help of recurrence plots and recurrence quantifications methods. The two cases of composite machining are analyzed in detail. The first case is a milling of undamaged material, while the second is a milling of damaged material. The detailed recurrence analysis shows interesting results, and additionally some of all quantifications can be used to damage detection.
\end{abstract}

Keywords Recurrence plots $\cdot$ Recurrence quantifications $\cdot$ Damage $\cdot$ Milling

\section{Introduction}

A composite material consist of two or more materials that results in better properties than when the individual components are used alone [2]. They are more difficult to machine than classical materials mainly because they are anisotropic,

The financial support of Structural Funds in the Operational Programme-Innovative Economy (IE OP) financed from the European Regional Development Fund Project "Modern material technologies in aerospace industry", No. POIG.01.01.02-00-015/0800 is gratefully acknowledged.

Krzysztof Kecik

k.kecik@pollub.pl

1 Lublin University of Technology, Nadbystrzycka 36, 20-618 Lublin, Poland nonhomogeneous, and are reinforced by hard glass or carbon fibers. Additionally, they are prone to damage: delamination, cracking, fiber pull-out, fiber fuzzing (especially aramid fibers), matrix chipping, and heat damage [1].

In many different applications of signal analysis is main way to track changes of a system's dynamics. One of the newer methods for time series analysis (efficient for short time series) is recurrence plot (RP). Recurrence is a fundamental property of dynamical systems, which can be exploited to characterize the system's behavior in phase space [22]. The recurrence plot is a new and modern versatile tool for the study of the complex behavior of dynamical systems. Recurrence is a fundamental property of dynamical systems, which can be exploited to characterize the system's behavior in phase space. Recurrence plots method, firstly was introduced by Eckmann et al. in 1987 [4]. Initially, this method was used for the purposes of visualization of system trajectories (especially in the higher dimensional phase spaces). Nowadays, the technique has been successfully applied to different fields ranging from physiology [15] to economics [6]. Based on RP method, the statistical analysis of their results was developed, called recurrence qualification analysis (RQA).

In paper [7], the feasibility of RQA to detect flank wear in face milling has been investigated. Both qualitative and quantitative approaches were used in order to detect tool wear. In the qualitative approach, the RP could make distinction between sharp and wear inserts based on the color patterns. RP was parametrized to give RQA parameters. Most of these parameters were able to show definite trend in RQA parameters with variation in insert wear. In general, recurrence rate, rapping time, laminarity, and entropy for showed definite trend and hence can be used 
in detecting insert wear in face milling. Litak et al. [8] investigated the time series of a torque applied to the ripping head in the process of cutting a concrete rock with sharp and blunt tools. By applying non-linear embedding methods and the recurrence plots technique to the corresponding time series, the changes in non-linear dynamics lying behind the ripping process were indicated. Additionally, authors proposed of using this technique for testing of the ripping machine efficiency and a method to monitor the state of tools.

Nichols et al. [9] have mentioned that RQA as a useful tool for detecting subtle non stationariness and/or changes in time series. Based on the structure found in recurrence plots, a variety of metrics are extracted including percentage of recurrence points, a measure reflecting determinism, and entropy. The approach is demonstrated experimentally in diagnosing the length of a crack in a thin steel plate. Gonzleza et al. [10] analyzed the electrochemical current oscillations generated during the early stages of corrosion fatigue damage using RPs and RQA and could separate the electrochemical process of pitting corrosion from the corrosion fatigue crack initiation and growth.

Kecik et al. [3] study the stability of a high-speed milling process of nickel super-alloy Inconel $713 \mathrm{C}$ by methods used in non-linear dynamics. Stability lobe diagram was a result of modal analysis and next verified by recurrence plots, recurrence quantification analysis, and classical non-linear methods. A stability lobes diagram shows the indistinct boundary between chatter-free stable machining and unstable processes. Sen et al. [11] studied the fluctuations of mean indicated pressure (MIP) in a diesel engine using recurrence plots, recurrence quantification analysis, and continuous wavelet transform. Sen has proposed the use of results of their study in developing effective control strategies for efficient engine performance.

Iwaniec et al. [23] study two aluminium plates: undamaged and cracked plate, excited to vibrations with the use of harmonic signals of frequencies corresponding to the frequencies of the first, third, and sixth system mode shapes. In the obtained results, it can be stated that the recurrence plots and recurrence quantification analysis methods can be used for detection of changes in the dynamic behavior (determinism) of mechanical systems related to changes in the frequency of excitation or crack propagation.

This paper presents the recurrence plots and recurrence quantification analysis that were used in detecting the differences in the dynamic behavior, for two cases of CFRP milling. The first test is a milling of undamaged material, while the second damaged. Carried out research made it possible to establish which of the RQA measures are most sensitive to changes in the dynamic properties that comes from damage appearance. The obtained results are first step to identification of damage during machining of CFRP material. All the necessary computations were performed with the use of functions implemented in the CRP Toolbox for MATLAB $[24,30]$.

\section{Recurrence plots and quantifications}

\subsection{Delay coordinate embedding}

In this section, we briefly outline some of the basic features and describe how one generates a recurrence plot. The first step to applied the recurrence technique is dynamics reconstruction by embedding the one-dimensional time series in $m$ dimensional reconstruction space using the delay coordinates (delay method). According to Takens theorem [34], it is possible to reconstruct a phase space trajectory from such a single scalar time series $\left(x_{1}, x_{2}, \ldots, x_{N}\right)$, where $N$ is the number of observed states. The reconstructed embedded vector $y(i)$ has form

$y(i)=\left(x_{i}, x_{i+d}, x_{i+2 d}, \ldots, x_{i+(m-1) d}\right)$,

where $d$ and $m$ are called, respectively, the time delay and the embedding dimension (called the embedding parameters). The embedding vector dimension can be defined as the minimum number dynamical variables that can describe the system attractor. Each unknown point of the phase space at time $i$ is reconstructed by the delayed vector $y(i)$ in an $m$-dimensional space called the reconstructed phase space (RPS). According to Eq. 1, the time is defined as

$t=i \Delta t$,

where $\Delta t$ is a sampling time.

The correct choice of proper delay parameters is a crucial matter. The most natural question pertains on how to choose an appropriate value for the time delay $d$ and the embedding dimension $m$. To calculate the parameter $d$, usually two method can be used. The first one is the value for which the autocorrelation function $C(d)$

$C(d)=\frac{1}{N-d} \sum_{i=1}^{N-d}\left(x_{i}-\bar{x}\right)\left(x_{i+d}-\bar{x}\right)$

first passes through zero is searched, which gives $d$.

The second method (often used) chooses the first minimum location of the average mutual information function, where the mutual information function is defined as follows

$S(d)=-\sum_{i j} p_{i j}(d) \ln \frac{p_{i j}(d)}{p_{i} p_{j}}$,

where $p_{i}$ and $p_{j}$ are the probability to find a time series value in the $i$ th and $j$ th interval of the partition, $p_{i j}(d)$ is the joint probability to find a time series value in the $i$ th 
interval and a time series value in the $j$ th interval after a time $d$ (the probability of transition in d time from the $i$ th to the $j$ th interval. The value $d$ that firstly minimizes the quantity $S(d)$ is the method choice for finding a reasonable time delay. Which method is "best," this is a very subjective.

The method used to find the embedding dimension is based on the concept of false neighbor. A false neighbor is a point in the data set that looks like a neighbor to another because the orbit is seen in a too small embedding space. In practice, the percentage of false nearest neighbors (FNN) is computed for each $m$ of a set of values; the embedding dimension is said to be found for the first percentage of FNN dropping to zero. Notice that when the signal is noisy, this percentage never reaches a true zero value.

\subsection{Recurrence plot diagram}

After the embedded vector reconstruction $y(i)$, the recurrence plot (RP) of a signal can be calculated. Recurrence plots are graphical structures elaborated by Eckmann, Kamphorst, and Ruelle in 1987 [4]. The recurrence diagram is a visualization of a square matrix, in which the points correspond to those times at which a state of a dynamical system recurs (reveals all the times, when the phase space trajectory of the dynamical system visits roughly the same area in the phase space). Practically, the recurrence plot is a symmetric graph that shows different structures mainly consisting of lines and points. In order to obtain the RP diagram, the $R \otimes R$ recurrence matrix, the elements of which can be computed using equation

$\mathbf{R}_{i, j}=H\left(\epsilon-\left\|x_{i}-x_{j}\right\|\right), i, j=1,2, \ldots n$,

where $n=N-(m-1) d, \epsilon$ is a predefined threshold distance, $\|$.$\| is the norm (in our study, the Euclidean norm was$ applied) and $H$ is the Heaviside function. This means that if two phase space vectors $x_{i}$ and $x_{j}$ are sufficiently close together, then $R_{i, j}=1$; otherwise, $R_{i, j}=0$.

The RP diagram is obtained by plotting results of Eq. 5 . The diagram is plotted in such a way that the distance between $x_{i}$ and $x_{j}$ is less than $\epsilon$, then a dot is placed. A crucial parameter of a RP is the threshold $\epsilon$. Therefore, special attention has to be required for its choice. If $\epsilon$ is chosen too small, there may be almost no recurrence points and we cannot learn anything about the recurrence structure of the underlying system. On the other hand, if $\epsilon$ is chosen too large, almost every point is a neighbor of every other point, which leads to a lot of artefacts. A too large includes also points into the neighborhood which are simple consecutive points on the trajectory [5]. Several criteria for the choice of the cut-off distance $\epsilon$ have been proposed [12,13].
The RP method is very useful for short time series. It allows finding different dynamics as periodic, drift, stationary random behavior, and even abrupt changes. The basic idea interpretation of the RP diagrams seems simple: if the underlying signal is truly random and has no structure, and so there will not be any identifiable patterns. If, on the other hand, the periodic signal can be detected by long diagonal line parallel to line of identity (LOI). Periodic and quasiperiodic systems mainly have RPs with diagonal oriented recurrent structures, mainly diagonal lines, checkerboard structures. While abrupt changes in the dynamics as well as extreme events cause white areas or bands in the RP. Additionally, the RP allows finding and assessing extreme and rare events easily by using the frequency of their recurrences.

\subsection{Recurrence quantification analysis}

The RP was able to provide only qualitative visual information. The following development of RP method introduced by Zbilut and Webber [14, 15], and later by Marwan et al. [16], has consolidated the RP method as a tool in nonlinear data analysis. They proposed a statistical description of recurrence plots and gave it the name of quantifications and called this technique recurrence quantification analysis (RQA). The quantities are based on points and lines. One of the quantities, the recurrence rate (RR), measures the ratio between recurrence points and all possible points on the graph.

The first of these statistics termed is recurrence rate (RR), which is the percentage of points of RP that are darkened. The RR is defined [5]

$\mathrm{RR}=\frac{1}{N^{2}} \sum_{i, j=1}^{N} \mathbf{R}_{i, j}^{m, \epsilon}$.

The next statistical quantification is the determinism (DET), defined as

$\mathrm{DET}=\frac{\sum_{l=l_{\min }}^{N} l P^{\epsilon}(l)}{\sum_{i, j=1}^{N} \mathbf{R}_{i, j}^{m, \epsilon}}$,

where $P^{\epsilon}(l)=\left\{l_{i} ; i=1 \ldots N_{l}\right\}$ is the frequency distribution of the lengths $l$ of diagonal structures and $N_{l}$ is the absolute number of diagonal lines. Note, in a periodic system, all points would be included in the lines.

The longest diagonal line, $L_{\max }$, measures the length of the longest diagonal line segment in the RP structure, excluding the main diagonal line.

$L_{\max }=\max \left(\left\{l_{i} ; i=1 \ldots N_{l}\right\}\right)$ or $\mathrm{DIV}=\frac{1}{L_{\max }}$. 
Fig. 1 The scheme of milling process of composite material with the artificial damage

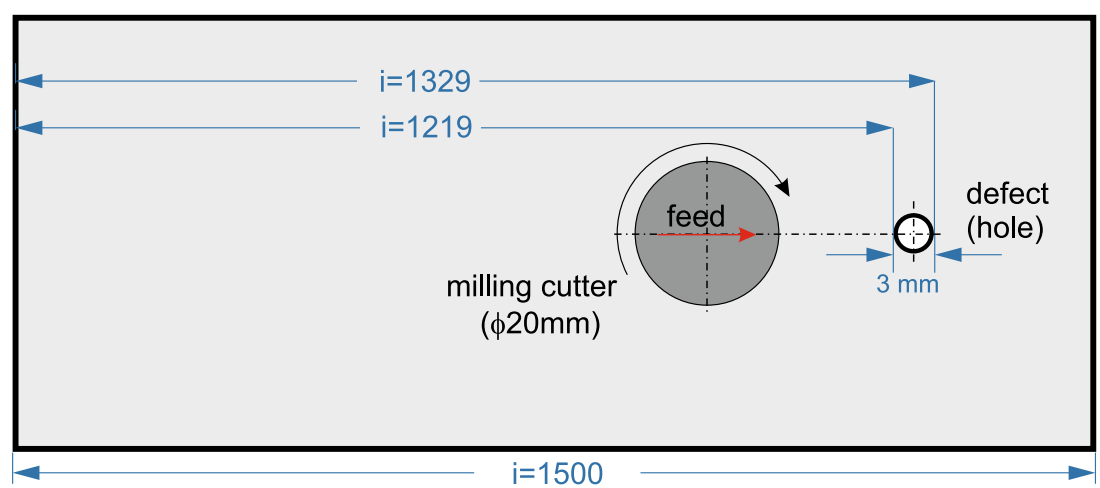

The $L_{\max }$ very often can be expressed as divergence (DIV). This parameter is a very important recurrence variable because it is inversely related to the largest positive Lyapunov exponent $\left(\right.$ Lyap $\left._{\max }\right)$. The positive Lyapunov exponents gauge the rate at which trajectories diverge and are the hallmarks for dynamic chaos. Thus, the shorter the $L_{\max }$, the more chaotic (less stable) the signal will be.

Similarly, the longest vertical line, $V_{\max }$, can be expressed

$V_{\max }=\max \left(\left\{v_{i} ; i=1 \ldots N_{v}\right\}\right)$.

The following statistical parameter is an entropy (ENTR). It is the Shannon entropy of the distribution of the length of diagonal segments

$\operatorname{ENTR}=-\sum_{l=l_{\min }}^{N} p(l) \ln p(l)$, where $p(l)=\frac{p^{\epsilon}(l)}{\sum_{l=l_{\min }}^{N} p^{\epsilon}(l)}$.

The $p(l)$ is the probability density of the diagonal structure length $l$. ENTR reflects the complexity of the RP in respect of the diagonal lines.

The ratio between recurrence points forming the vertical structures and the entire set of recurrence points (the percentage of recurrence points which form vertical lines) called laminarity (LAM) and computed

$\mathrm{LAM}=\frac{\sum_{v=v_{\min }}^{N} v p^{\epsilon}(v)}{\sum_{v=v_{1}}^{N} v p^{\epsilon}(v)}$,

where $p^{\epsilon}(v)=\left\{v_{i} ; i=1 \ldots N_{v}\right\}$ denotes the frequency distribution of the lengths $l$ of the vertical structures.

The definition of the average length of the vertical structures called trapping time (TT)

$\mathrm{TT}=\frac{\sum_{v=v_{\min }}^{N} v p^{\epsilon}(v)}{\sum_{v=v_{\min }}^{N} p^{\epsilon}(v)}$.

The computation also uses the minimum length $v_{\min }$ as in LAM, Eq. 11.
Gao and Cai [17] in 2000 introduced a recurrence time of first $T 1$ and second $T 2$ type

$\left.\left.T_{1}=\mid\left\{i, j: \overline{x_{i}}, \overline{x_{j}}\right\} \in R_{i}\right\}\left|, \quad T_{2}=\right|\left\{i, j: \overline{x_{i}}, \overline{x_{j}}\right\} \in R_{i} ; \overline{x_{j}} \notin R_{i}\right\} \mid$,

where $R_{i}$ are the recurrence points which belong to the state $\overline{x_{i}}$. Marwan shows that $T 2$ is able to detect weak transitions with high accuracy, both in clean and noisy environment [18].

The distribution $H_{v}(v)$ can applied to derive recurrence time entropy (RTE), called recurrence period density entropy (RPDE).

$\mathrm{RPDE}=-\frac{1}{\ln V_{\max }} \sum_{v=1}^{V_{\max }} H_{v}(v) \ln H_{v}(v)$

This measure quantifies the extent of recurrences and is related to the Pesin dimension (PD) [19].

Recently, Marwan et al. [21] proposed a new quantifications based on complex network theory, as clustering coefficient $(\mathrm{CC})$

$\mathrm{CC}=\sum_{i=1}^{N} \frac{\sum_{j, k=1}^{N} \mathbf{R}_{i, j}^{m, \epsilon} \mathbf{R}_{j, k}^{m, \epsilon} \mathbf{R}_{k, i}^{m, \epsilon}}{\mathrm{RR}_{i}}$,

where $\mathrm{RR}_{i}=\sum_{j=1}^{N} \mathbf{R}_{i, j}^{m, \epsilon}$ the local recurrence rate, $k$ is defined as the number of neighbors.

The second recurrence quantifications is called transitivity (TRANS) and calculated from equation

TRANS $=\frac{\sum_{i, j, k=1}^{N} \mathbf{R}_{i, j}^{m, \epsilon} \mathbf{R}_{j, k}^{m, \epsilon} \mathbf{R}_{k, i}^{m, \epsilon}}{\sum_{i, j, k=1}^{N} \mathbf{R}_{i, j}^{m, \epsilon} \mathbf{R}_{k, i}^{m, \epsilon}}$.

Regular dynamics usually is associated with a higher transitivity, while chaotic systems show comparatively low values [20].

Generally, the RP and RQA techniques are used to detecting changes in data behavior, in particular in detecting breaks, like a phase transition and in informing about other dynamic properties of a time series. The RP and RQA 
Fig. 2 The normalized cutting force $F_{x}$ responses of composite machining for milling without (a) and artificial defect (b)

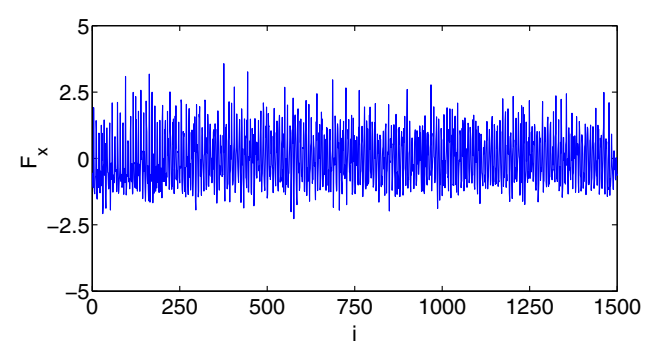

(a) $F_{x}$

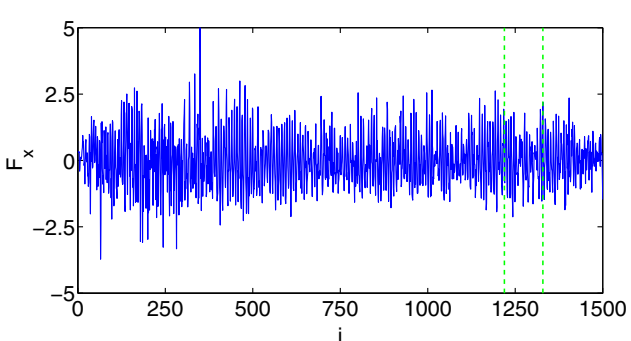

(b) $F_{x}$ methods can be also used to provide evidence of chaos and also can used for they appropriateness in working in nonstationarity and noisy data [29].

\section{Time series analysis based on recurrence plots}

\subsection{Experiment methodology}

The carbon fiber reinforced plastic (CFRP) machining were conducted on the vertical machining center Avila-VMC $800 \mathrm{HS}$. The cutting tool has a two-coated diamond inserts (PCD) about diameter $\phi=20 \mathrm{~mm}$, produced by Kennametal (with symbol EC1004FR PCD-1410 KD). The tests have been carried on CFRP, saturated with epoxy resin (trade name GR/EP 985). The cutting forces were measured using the 3D Kistler dynamometer (type 9257B) connected to multichannel charge amplifier (type 5070). The measured signals were saved by software Dynowave (type 2825A) with Data Acquisition Card Dynoware (type 5697A).

Machining of CFRP was performed for fixed cutting parameters: speed $V_{c}=250 \mathrm{~m} / \mathrm{min}$, axial depth of cut $a_{p}=1 \mathrm{~mm}$, radial depth of cut $a_{e}=20 \mathrm{~mm}$, feed $f_{z}=0.2 \mathrm{~mm} /$ edge. The sampling time was fixed and equals $\Delta t=0.001 \mathrm{~s}$. The scheme of machining of composite material with the artificial damage in Fig. 1 is shown. The damage was located between 1219 and 1329 data points, then the total time of pass by damage equals $0.11 \mathrm{~s}$.
In order to recurrence analysis, the cutting force $F_{x}$ have been chosen. This force is oriented in the feed direction. Because of comparing two different experimental tests, the normalization of data have been applied, as suggest in paper [21]. Each time series was subjected to the process of normalization of data by classical method: normalizes the signal to mean $\left(^{-}\right)$and standard deviation $(\sigma)$ one.

$F_{x}(i)=\frac{F_{X}(i)-\overline{F_{X}}}{\sigma\left(F_{X}\right)}$.

The time history of cutting forces, for composite machining material without damage in Fig. 2a, and with damage in Fig. 2b, are shown. The green line in Fig. 2b denotes position of damage in composite material. Note, that these figures show full machining: i.e., the entry and exit tool from machining material were rejected. Comparing both time series, we can conclude that the courses are similar. However, for machining material with damage, the measured signal included more stochastic components and envelope of a curve is more uneven. Probably, this is caused by tool wear and change dynamics comes from placing the damage.

The detection of damage by RP method has a few assumptions. The main assumption is that damage should be the appropriate size and depth. In our study, we assumed that damage equals depth of cut, but the diameter ratio between damage and tool is 0.15 . Additionally, the sampling of the signal must be high especially for small diameter and high
Fig. 3 The mutual information (a) and false neighbors method (b) for estimation of parameters $d$ and $m$

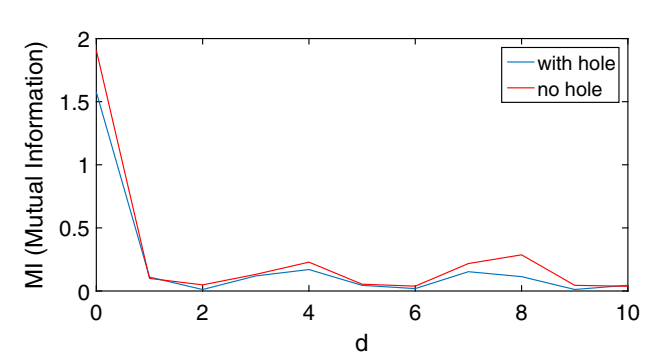

(a)

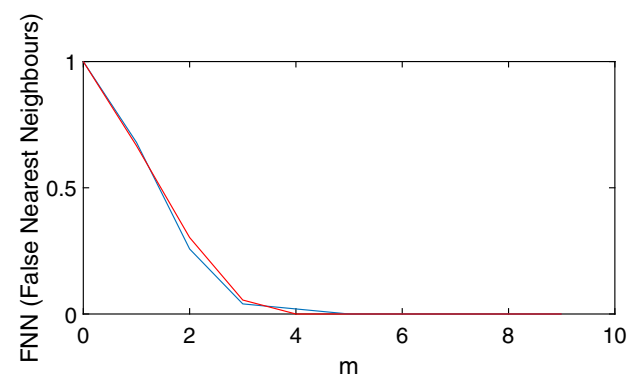

(b) 
Fig. 4 Recurrence plots determined for $F_{x}$ cutting for milling test without (a) and with damage (b), for $m=6, d=2, \epsilon=1.5 \sigma$, and L2 norm

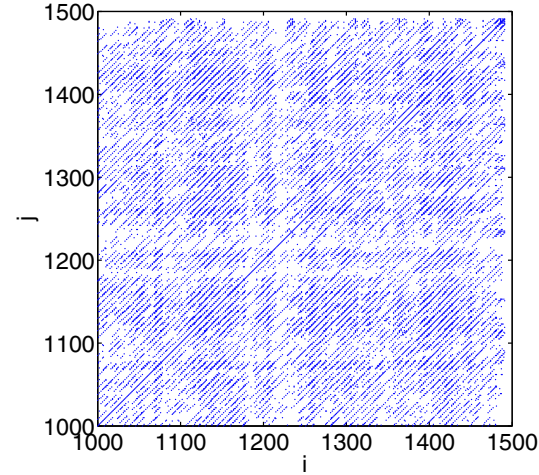

(a)

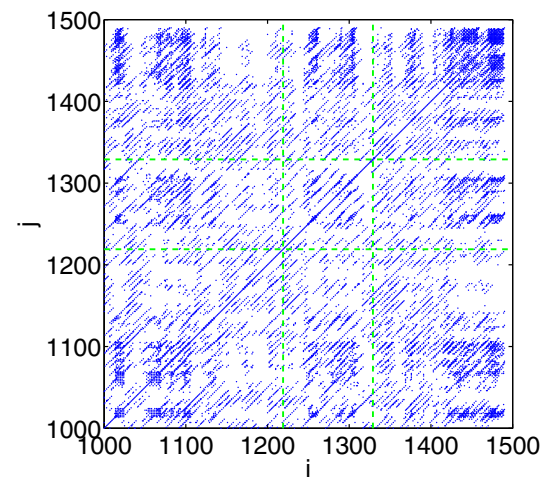

(b) cutting speed. The second problem of the method is choosing proper value of the indexes used to damage detection. In this aim, several experimental tests should be done.

\subsection{Recurrence plots of experimental data}

In order to estimate the recurrence plots and quantifications, the delay parameters $d$ and $m$ have been calculated by method presented in Section 2.1. The result in Fig. 3a shows that optimal value (the first minimum) of delay parameters has value $d=2$ for both cases. Similar results have been obtained for embedding delay, where the same value of $m=6$ was obtained for both tests. Once the $m$ and $d$ parameters have been estimated, the recurrence plots can be computed. The results for $F_{x}$ cutting forces are given in Fig. 4. The RP diagram for milling composite material without damage in Fig. $4 \mathrm{a}$ is presented.

The reconstructed recurrence plots were calculated in $L 2$ norm, called Euclidean. The norm is by far the most commonly used norm for RP method. The location of the damage is denoted by green line. The plot mainly consist of lines and points. To analyze, five hundred points $(0.5 \mathrm{~s})$ were taken. However, for material milling with damage, the RP diagram is more complicated, the blacked and "empty" areas appeared. This probably is caused by introduced damage which changes the stiffness and dynamics tool-workpiece system. Both experimental tests were made by the same tool, therefore its wear little could affect. Based on the results of RP diagrams, it is very difficult to determine the location of damage. Therefore, RPs require more precisely advanced analysis-RQA.

\subsection{Detection of damage by RQA method}

In order to go beyond the visual impression yielded by RPs, several measures of complexity which are calculated. All recurrence quantifications are calculated using moving window technique. First, the widows size was chosen $n i=50$ points, and quantifications were calculated; next, the widow moves about window step vale $\Delta i=1$ and calculations were repeated. This method allows observing and detecting the sudden dynamical changes, which can be caused by damage or tool wear.

The first calculated quantification was recurrence rate (RR), (Fig. 5), which generally characterizes density of recurrence points in RP diagram. This qualification shows change of dynamics (especially for material with damage, Fig. 5b), but identification of damage (hole) by it seems rather difficult. However, between two green lines (in hole), the RR slightly changes value, initially increases and next decrees (the cutting resistance initially decreases if the tool begins cut in hole and next increases). After exit, the tool with hole machining, RR rapidly increases (probably caused by smaller cutting force, see Fig. 2b) and vibrations.

The next analyzed quantification is a determinism (DET), which is the fraction of recurrence points forming diago-
Fig. 5 Change of RR for test without (a) and with damage (b). The RQA parameters are: $\Delta i=1, n i=50$

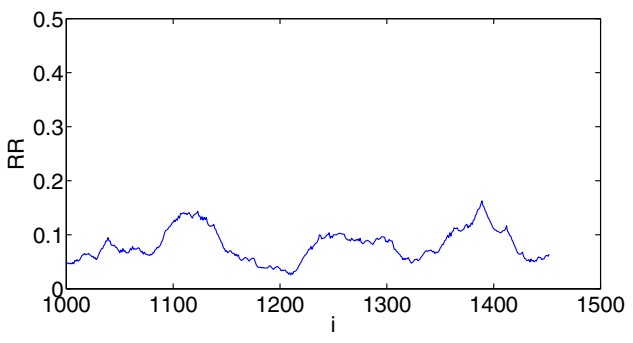

(a)

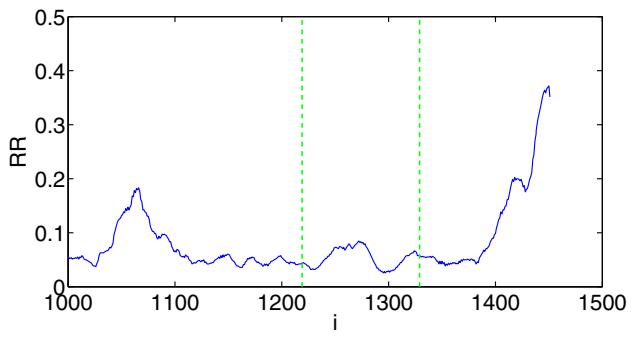

(b) 
nal lines. Interestingly, that DET, for milling of "health" material rapidly changes values between 0 and 0.65 , which maybe due by variable cutting resistance (comes from fibers orientation); see Fig. 6 a.

Similar results is observed for machining of "hole material", however, if the tool enter in the hole region (between two green lines), then the value of DET is stabilizing near the value of $0.35-0.4$. This suggest that DET shows dynamic changes during machining material with "hole", what can be used to detect of damage.

The following analyzed quantifications are longest diagonal (Fig. 7) and vertical (Fig. 8) lines. The $L_{\max }$ is simply the length of the longest diagonal line segment in the recurrence diagram (excluding LOI). Of course, $V_{\max }$ is defined similarly as simply the length of the longest vertical line segment. Analyzing results of Figs. 7 and 8, it shows that $V_{\max }$ can be used as detector of damage. The value of $V_{\max }$ is changed for "hole" milling. Unfortunately, other changes dynamics of machining for $i=1000 \div 1100$ and $i=$ $1350 \div 1400$ were detect, also. Therefore, the main problem of applied $V_{\max }$ as damage indicator is how to choose its value. The answer to this question requires a lot of further research and analysis.

The next analyzed recurrence variable are entropy (ENTR), laminarity (LAM), and trapping time (TT), which attention is focused on vertical line structures and black patches. For the detection of chaos-chaos transitions, Marwan [16] proposes using laminarity and trapping time. Generally, TT characterizes the average length of vertical line structures. It represents the average time in which the system is "trapped" in a specific state. LAM measures the percentage of recurrent points comprising vertical line structures. The entropies refers to the Shannon entropy of the distribution probability of the diagonal lines length. The change of ENTR, LAM, and TT are shown in Figs. 9, 10, and 11 , respectively. When analyzing the changes in the value of quantifications, it seems that the best to detect of hole is trapping time. This parameter practically has stable value (equals 2), but then dynamics is changed, then TT increases (Fig. 11b). Additionally, for milling undamaged material, this parameter is stable equals TT $=2$ (Fig. 11a).
Analyzing the obtained results, we can conclude that introducing hole in machining material can change the dynamics of process milling not only in the hole region but also in its vicinity. The change of entropy disallows observing any symptoms, which can be used to detect the damage (Fig. 9a, b). The same situations with variable LAM, it value rapidly changes in all analyzed time region and difficult damage identify.

The change of quantification parameters recurrence time of first type (T1) and second type (T2) are presented in Figs. 12 and 13, repetitively. Both recurrence times describe the time distances between the state at time $i$ and $j$ [25]. Obtained recurrence quantifications show change in dynamics during milling of damaged material. The value of $T 1$ and $T 2$ distinctly increases and receive maximal value (about 20). Note, that the recurrence time diagrams $T 1$ and $T 2$ are very similar.

Interestingly, change of recurrence period density (RPDE) (or recurrence time entropy, RTE) in Fig. 14a, b is shown. In practice, RPDE has the ability to detect subtle changes in time series such as the breakdown of regular periodic oscillation which usually are hard to detect using classical signal processing tools (e.g., Fourier transform). In hole region, the value of RPDE receive maximal and minimal values. The recurrence time entropy can be used to detect damage, too.

The two last analyzed recurrence quantifications are clustering coefficient (CC, Fig. 15) and transitivity (TRANS, Fig. 16). In literature, the TRANS can distinguish regular from irregular dynamics [20, 25, 27], and thus, reveals the periodic window and the unstable periodic orbit (UPO) due to the more regular dynamics than during the other chaotic regimes. The clustering coefficient characterize the density of connections among the neighbors [20].

It is frequently referred to as network transitivity [26, 28]. Additionally, the CC is a good indicator for the presence of periodic dynamics [20]. Generally, the TRANS and especially CC are not recommended to detect damage (Figs. 15b and 16b). However, the TRANS shows some symptom, which can inform about changes in dynamics of process. The variable CC practically does not show anything. The RP
Fig. 6 Change of DET for test without (a) and with damage (b). The RQA parameters are: $\Delta i=1, n i=50$

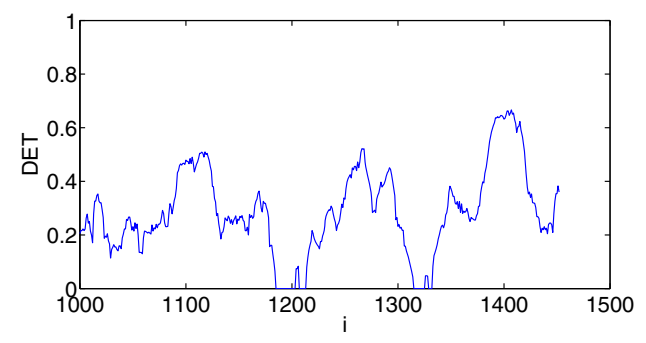

(a)

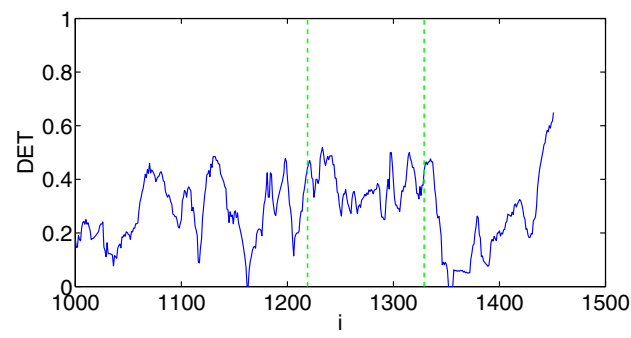

(b) 
Fig. 7 Change of $L_{\max }$ for test without (a) and with damage (b). The RQA parameters are $\Delta i=1$ and $n i=50$

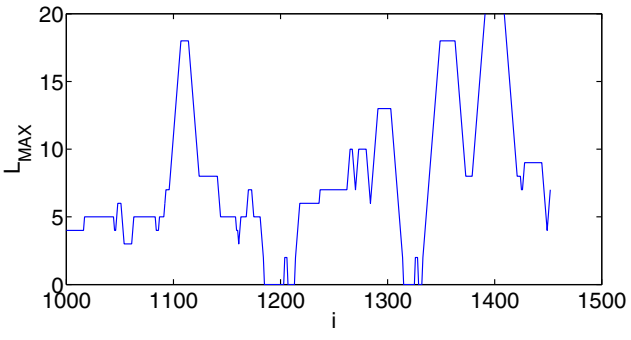

(a)

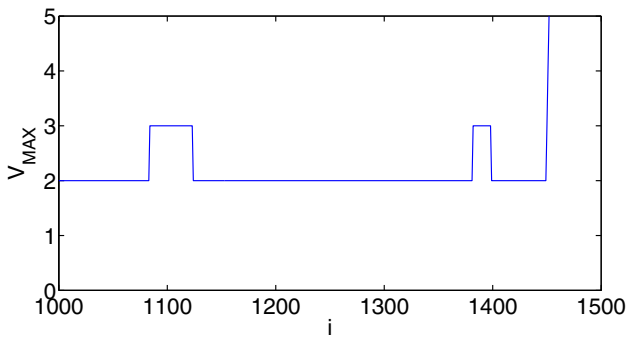

(a)

Fig. 9 Change of ENTR for test without (a) and with damage (b). The RQA parameters are $\Delta i=1$ and $n i=50$

Fig. 8 Change of $V_{\max }$ for test without (a) and with damage (b). The RQA parameters are $\Delta i=1$ and $n i=50$

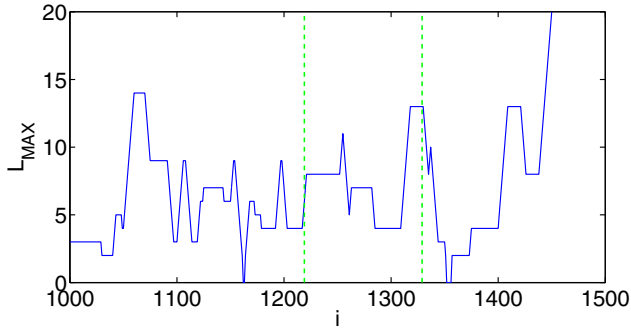

(b)

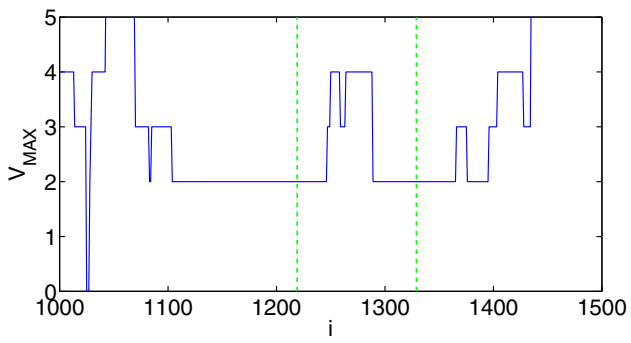

(b)

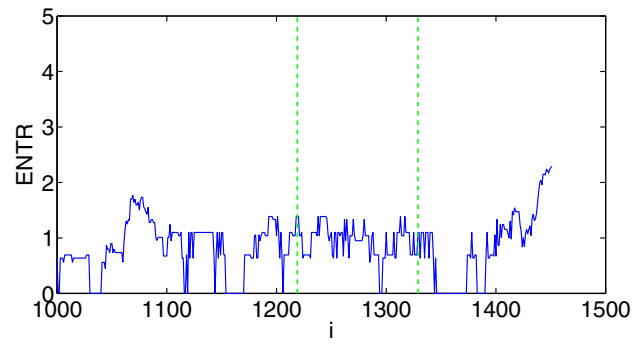

(b)

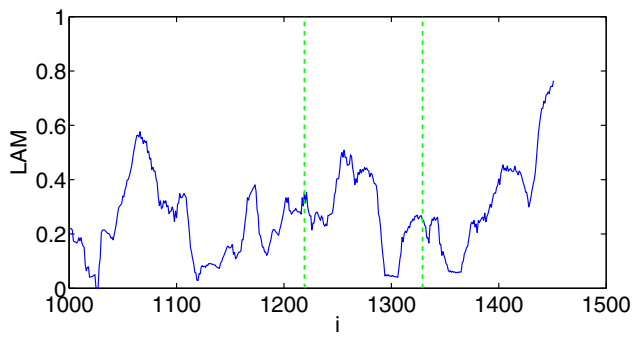

Fig. 11 Change of TT for test without (a) and with damage (b). The RQA parameters are $\Delta i=1$ and $n i=50$

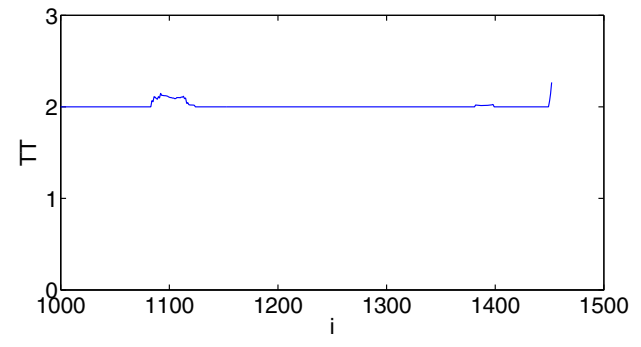

(a) (b)

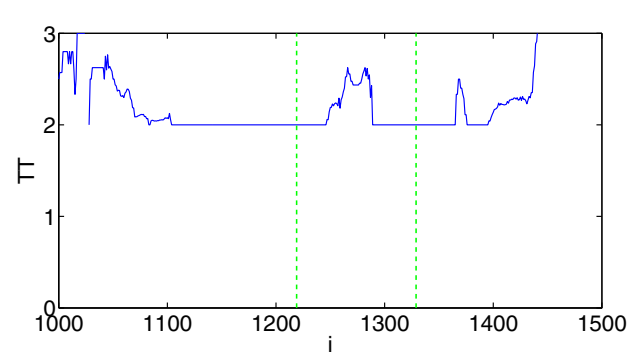

(b)

(a) 
Fig. 12 Change of $T 1$ for test without (a) and with damage (b). The RQA parameters are $\Delta i=1$ and $n i=50$

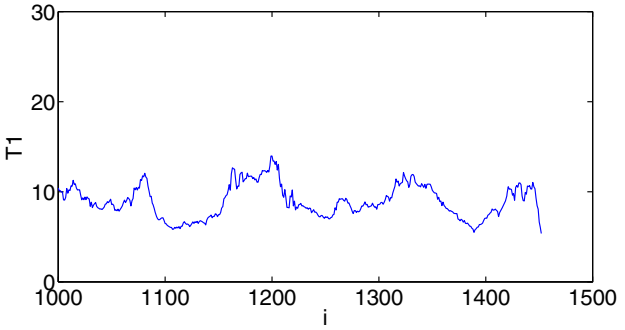

(a)

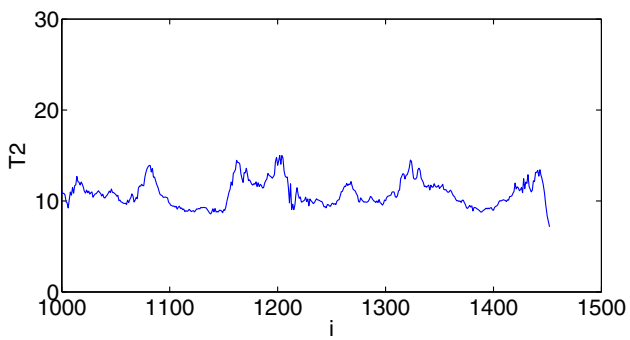

(a)

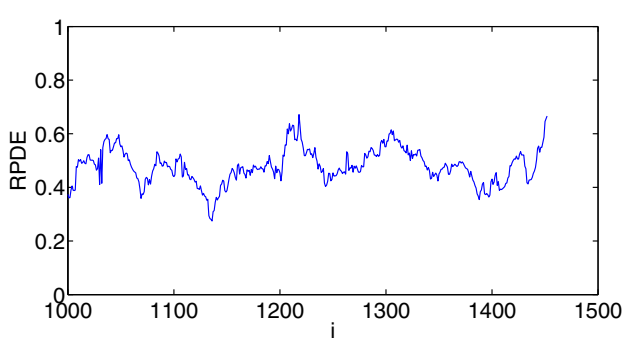

(a)

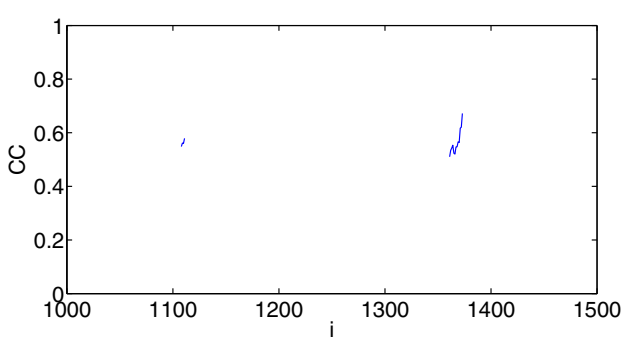

(a)

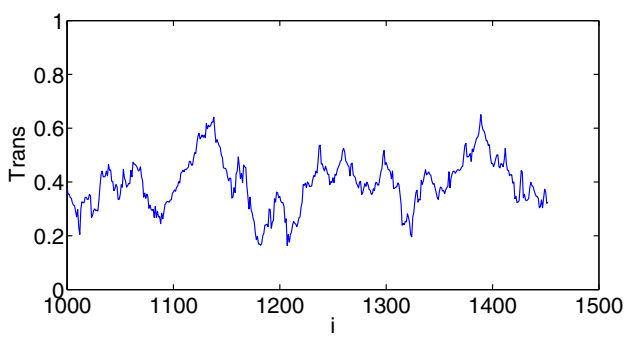

(a)

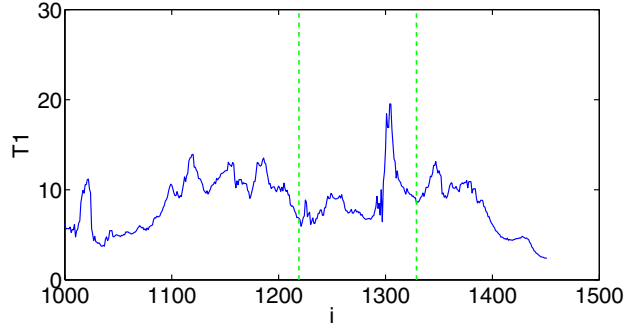

(b)

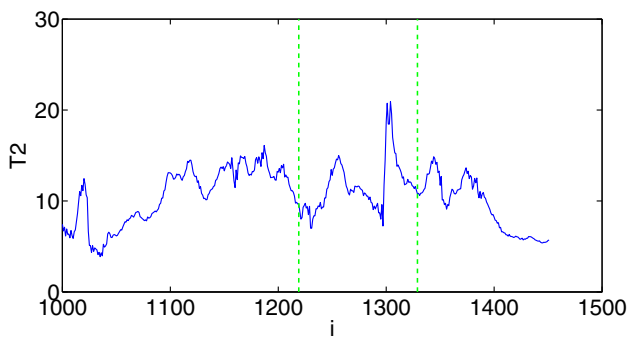

(b)

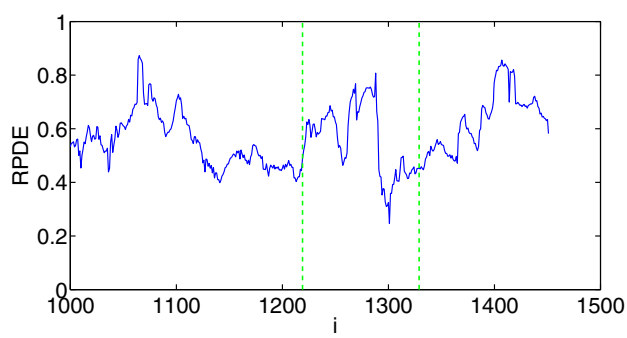

(b)

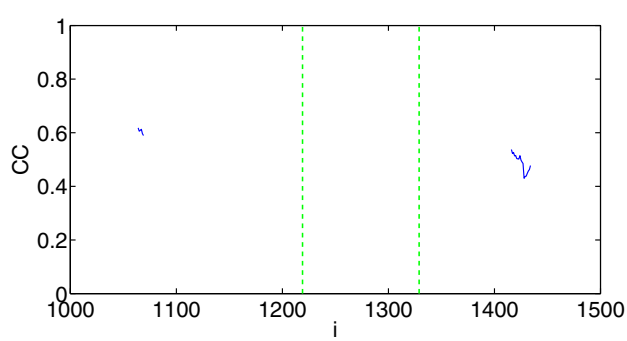

(b)

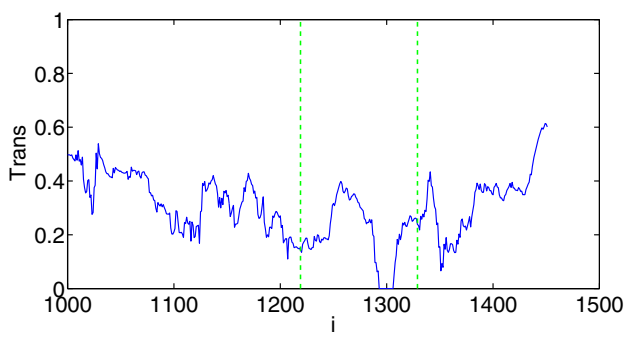

(b) 
Fig. 17 Selected RQA results made for the cutting force $F_{x}$ and for $m=5, d=2, \epsilon=1.5 \sigma$, and applied $L 2$ norm

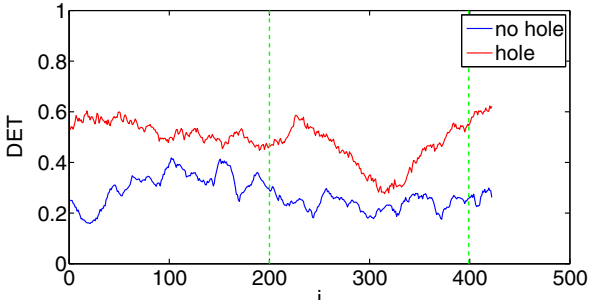

(a)

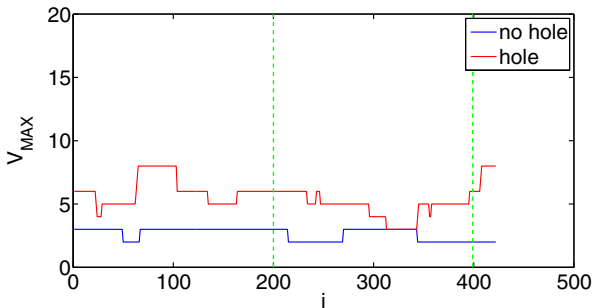

(c)

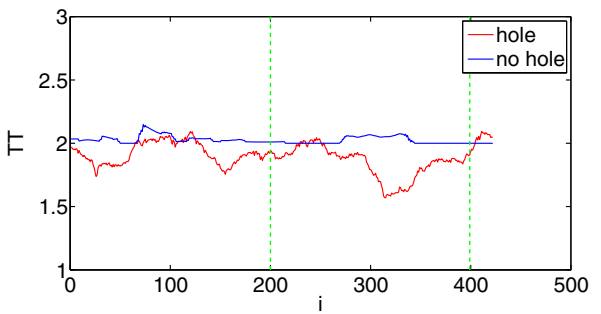

(e)

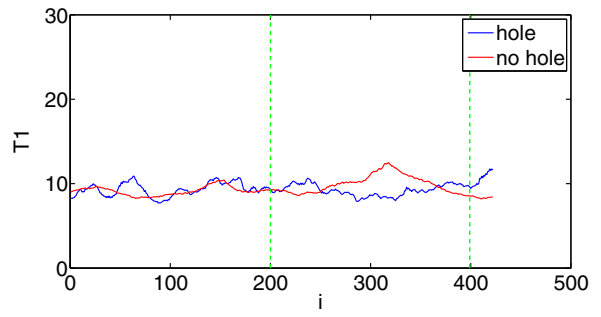

(g)

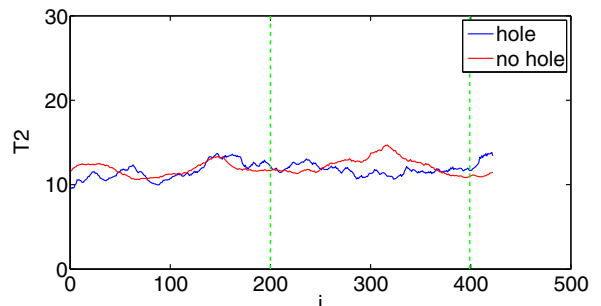

(i)

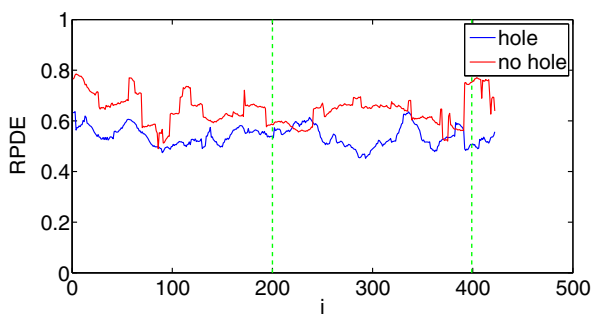

(k)

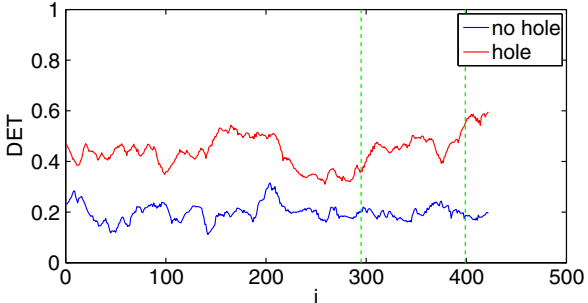

(b)

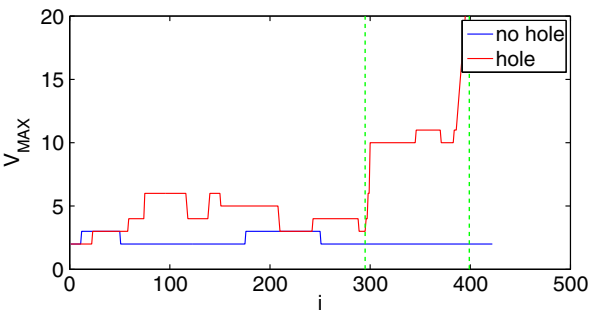

(d)

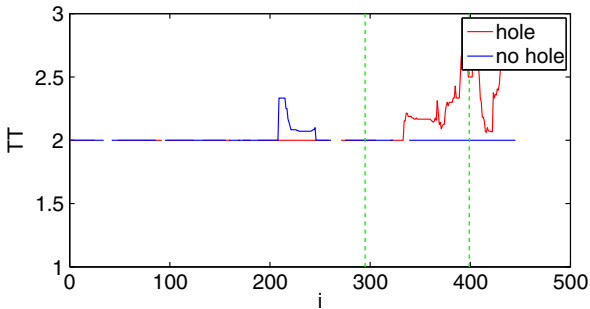

(f)

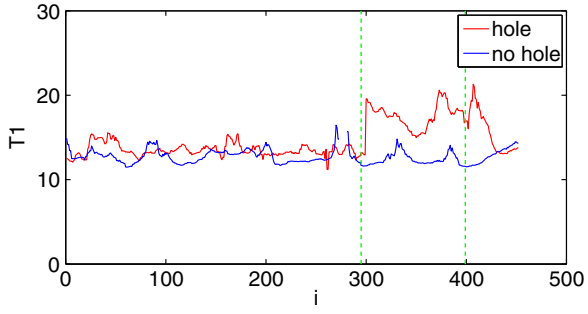

(h)

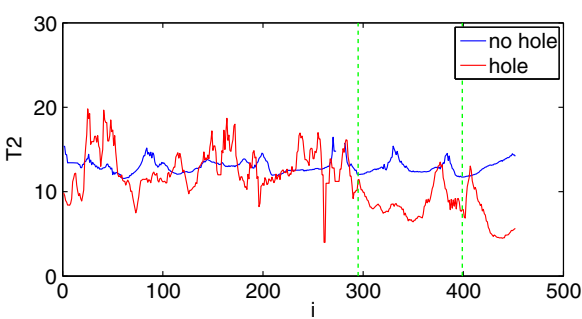

(j)

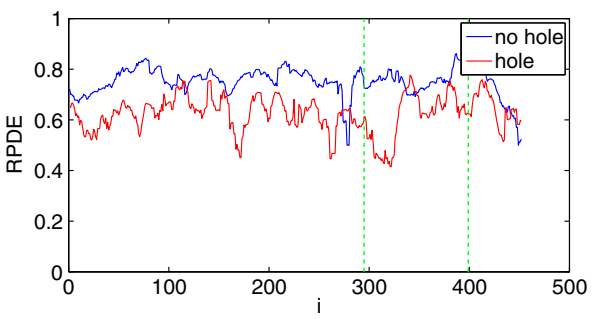

(1) 
and RQA analysis shows that some quantifications can be applied to detect of damage during machining of composite materials. This is very important from a practical point of view. Of course, this requires further research and analysis. Interestingly, that damage (hole) formed in the workpiece change cutting dynamic both it and in its vicinity.

Below, the recurrence quantification analysis (quantifications selected earlier) for other milling tests have been presented. The four additional tests have been presented. The size of hole and milling material, tool, and machining center have been identical as previously. The first test have been done for milling feed $f_{z}=0.1 \mathrm{~mm} / \mathrm{edge}$ (two times smaller compare to previously results) and cutting speed $V_{c}=250 \mathrm{~m} / \mathrm{min}$. The results are presented in figures: Fig. $17 \mathrm{a}, \mathrm{c}, \mathrm{e}, \mathrm{g}, \mathrm{i}, \mathrm{k}$.

The other results (Fig. 17b, d, f, h, j, l) present analysis for milling of the composite material with the feed $f_{z}=0.2 \mathrm{~mm} /$ edge and the higher cutting speed $V_{c}=350 \mathrm{~m} / \mathrm{min}$. The green line means position of the hole in material. The blue line presents tests for the undamaged material, while the red line denotes the RQA results for material with the damage. Analyzing the obtained results, we can conclude that it is possible to detect the artificial damage where the ratio between the damage and the tool diameter equals about .15. However, to properly identify the damage, it is best to use several indicators. Note, that authors have done more experimental tests and RQA analysis; however, the obtained results were similar.

\section{Conclusions and final remarks}

The paper concerns of dynamics analysis of cutting force components by means of the recurrence plots and recurrence quantification analysis methods. The proposed methods, due to their efficiency, versatility, and possibility of use for short signals, are nowadays commonly applied to analysis of non-linear data from various fields of research.

The RP were designed to detect to nonstationarity in time series; therefore, we think that it can be used for damage detection for milling process, too. Through laboratory experiments, it is proven that some quantifications can be uses as damage indicators. In order to select and verify quantifiers, the two cutting test with high cutting speed $\left(V_{c}=250 \mathrm{~m} / \mathrm{min}\right)$ were performed. The first test based on the milling of "health" carbon composite material. The second test was made for the milling of damage material. The damage was introduced artificially as hole. The ration between tool and damage (hole) equals about 7 , which had corresponded to natural damage occurring during machining.

The obtained result shows that the best recurrence quantifications to detect damage are determinism (DET), longest vertical line $\left(V_{\max }\right)$, trapping time (TT), recurrence times ( $T 1$ and $T 2$ ), and recurrence time entropy (RPDE). The main problem is how to estimate value of proposed quantifications? This requires a lot of further research and analysis.

The most promising seems trapping time which detects location of damage quite well. For milling of "health material, the TT has stable value equal to 2 . For a milling material with damage, the TT detects hole and other dynamics behavior. This probably is caused by tool wear, noise, and change of cutting resistance in the vicinity of hole (the fibers of composite material are broken and cut by the hole formation).

However, based on additional experimental tests, the application of several recurrence indicators seems promising. This way should be improving this method. The next step will be a detailed analysis of proposed recurrence qualifications for other cutting parameters and kinds of materials. The future work is application of the RP method to detect much smaller defects. Additionally, the implementation to real machining process to automatic detection is planned. The other method of recurrence plot joint recurrence plot (JRP) will be also tested.

Acknowledgments Financial support of Structural Funds in the Operational Programme Innovative Economy (IE OP) financed from the European Regional Development Fund-Project No POIG.0101.02-00-015/08 is gratefully acknowledged.

Open Access This article is distributed under the terms of the Creative Commons Attribution 4.0 International License (http://creativecommons.org/licenses/by/4.0/), which permits unrestricted use, distribution, and reproduction in any medium, provided you give appropriate credit to the original author(s) and the source, provide a link to the Creative Commons license, and indicate if changes were made.

\section{References}

1. Campbell FC (2010) Machining and assembly Structural composite material. ASM International, pp 307-332

2. Kecik K, Rusinek R, Warminski J, Weremczuk A (2012) Chatter control in the milling process of composite materials. J Phys Conf Ser 382:1-7

3. Kecik K, Rusinek R, Warminski J (2011) Stability lobes analysis of nickel superalloys milling. International Journal Bifurcation and Chaos 21(10):1-12

4. Eckmann JP, Kamphorst SO, Ruelle D (1987) Recurrence plots of dynamical system. Europhysics Letter 4:973-977

5. Marwan N, Romano MC, Thiel M, Kurths J (2007) Recurrence plots for the analysis of complex systems. Phys Rep 438:237-329

6. Belaire-Franch J (2004) Testing for Non-Linearity in an artificial financial market: a recurrence quantification approach. J Econ Behav Organ 54(4):483-494

7. Mhalsekar SD, Rao SS, Gangadharan KV (2010) Investigation on feasibility of recurrence quantification analysis for detecting flank wear in face milling. Int J Eng Sci Technol 2(5):23-38 
8. Litak G, Gajewski J, Syta A, Jonak J (2008) Quantitative estimation of the tool wear efects in a ripping head by recurrence plots. J Theor Appl Mech 46(3):521-530

9. Nichols JM, Trickey ST, Seaver M (2006) Damage detection using multivariate recurrence quantification analysis. Mech Syst Signal Process 20:421-437

10. Gonzleza NA, Ochoa EG, Snchez JG (2008) Assessment of the dynamics of corrosion fatigue crack initiation applying recurrence plots to the analysis of electrochemical noise data. Int $\mathbf{J}$ Fatigue 30(7):1211-1219

11. Sen AK, Longwic R, Litak G, Gorski K (2008) 2008 Analysis Of cycle-to-cycle pressure oscillations in a diesel engine. Mech Syst Signal Process 22(2):362-373

12. Matassini L, Kantz H, Holyst J, Hegger R (2002) Optimizing of recurrence plots for noise reduction. Physical review. E, Statistical, nonlinear, and soft matter physics 65:021102

13. Thiel M, Romano MC, Kurths J, Meucci R, Allaria E, Arecchi FT (2002) Influence of observational noise on the recurrence quantification analysis. Physica D 171:138-52

14. Zbilut JP, Webber CL Jr (1992) Embeddings and delays as derived from quantification of recurrence plots. Phys Lett A 171(3-4):199-203

15. Webber CL Jr, Zbilut JP (1994) Dynamical assessment of physiological systems and states using recurrence plot strategies. J Appl Physiol 76(2):965-973

16. Marwan N, Wessel N, Meyerfeldt U, Schirdewan A, Kurths J (2002) Recurrence Plot Based Measures of Complexity and its Application to Heart Rate Variability Data. Physics Review E 66(2):026702

17. Gao J, Cai H (2000) On the structures and quantification of recurrence plots. Phys Lett A 270:75-87

18. Marwan N, Riley M, Giuliani A, Webber ChL Jr (2014) Translational recurrences: From mathematical theory to Real-World applications. Springer, New York, pp 109-124
19. Marwan N, Kurths J, Foerster S (2015) Analysing spatially extended high-dimensional dynamics by recurrence plots. Phys Lett A 379:894-900

20. Webber ChL Jr, Marwan N (2015) Recurrence quantification analysis: Theory and best practices. Springer, London, pp 349 374

21. Marwan N, Donges JF, Zou Y, Donner RV, Kurths J (2009) Complex network approach for recurrence analysis of time series. Phys Lett A 373(46):4246-4254

22. Marwan N, Kurths J (2002) Nonlinear analysis of bivariate data with cross recurrence plots. Phys Lett A 302:299307

23. Iwaniec J, Uhl T, Staszewski WJ, Klepka A (2012) Detection of changes in cracked aluminium plate determinism by recurrence analysis. Nonlinear Dyn 70(1):125-140

24. Marwan N (2013) Cross Recurrence Plot Toolbox for Matlab, Reference Manual, Version 5.17, Release 28.17, http://tocsy. pik-potsdam.de

25. Marwan N (2003) Encounters With Neighbours - Current Developments Of Concepts Based On recurrence plots and their applications, Ph.D. Thesis. University of Potsdam

26. Shockley K, Butwill M, Zbilut J, Webber C (2002) Cross recurrence quantification of coupled oscillators. Phys Lett A 305:5969

27. Zou Y, Donner RV, Donges JF, Marwan N, Kurths J (2010) Identifying complex periodic windows in continuous-time dynamical systems using recurrence-based methods. Chaos 20(4):043130

28. Boccaletti S, Latora V, Moreno Y, Chavez M, Hwang DU (2006) Complex networks: Structure and dynamics. Physics Report 424(4-5):175-308

29. Chakrabarti BK, Chakraborti A, Chatterjee A (2006) Econophysics and Sociophysics: Trends and perspectives. WILEY-VCH, Weinheim

30. http://tocsy.pik-potsdam.de/CRPtoolbox/ 